Erratum

\title{
ERRATUM: Macrozoobenthos in an altitudinal gradient in North Patagonian Cautín River (Araucanía Region, Chile)
}

D. Figueroa ${ }^{\mathrm{a}^{*}}$ (1) and P. De los Rios-Escalante $\mathrm{b}^{\mathrm{b}, \mathrm{c}}$ (D)

aUniversidad Católica de Temuco, Facultad de Recursos Naturales, Departamento de Ciencias Ambientales, Casilla, Temuco, Chile bUniversidad Católica de Temuco, Facultad de Recursos Naturales, Departamento de Ciencias Biológicas y Químicas, Casilla, Temuco, Chile

'Núcleo de Estudios Ambientales UC Temuco, Casilla, Temuco, Chile

Due to author's honest mistake the article "Macrozoobenthos in an altitudinal gradient in North Patagonian Cautín River (Araucanía Region, Chile)" (DOI https://doi.org/10.1590/1519-6984.240484), published in Braz.J. Biol., vol. 82, São Carlos 2022, Epub: e240484, was published with an error.

On page 5 , where the Table 2 shows:

Udamocercia sp.

Pictetoperla gayi (Pictet, 1841)

Insecta, Plecoptera

Diamphipnoa sp.

It should show:

Andesiops peruviana (Ulmer, 1920)

Dactylobaetis sp.

Insecta, Plecoptera

Kaplopteris kuscheli, Illies, 1960.

The authors apologize for the errors. 